prevent in-hospital secondary transmission to other HCWs and inpatients. The concern of transmitting infection into the healthcare system has been highlighted in a recent study in which HCWs were linked to transmission of COVID-19 into long-term healthcare facilities. ${ }^{8}$ Considering that as many as $50 \%$ of all SARS-CoV-2 infections are asymptomatic, ${ }^{9}$ it would seem appropriate, when resources are available, to perform routine SARS-CoV-2 nasopharyngeal screening for all HCWs. The prediction model described by Tostmann et al, ${ }^{2}$ rather than fever and ARI symptoms, can be used to guide a targeted screening strategy in settings with limited availability of testing materials.

Acknowledgments. None.

Financial support. No financial support was provided relevant to this article.

Conflicts of interest. All authors report no conflicts of interest relevant to this article.

\section{References}

1. Kluytmans M, Buiting A, Pas S, et al. SARS-CoV-2 infection in 86 healthcare workers in two Dutch hospitals in March 2020. MedRxiv 2020. doi: 10.1101/ 2020.03.23.20041913.
2. Tostmann A, Bradley J, Bousema T, et al. Strong associations and moderate predictive value of early symptoms for SARS-CoV-2 test positivity among healthcare workers, the Netherlands, March 2020. Euro Surveill 2020;25:2000508.

3. Lo D. COVID-19: protecting healthcare workers. Lancet 2020;395:922.

4. Cheng V, Wong S-C, Chen J, et al. Escalating infection control response to the rapidly evolving epidemiology of the coronavirus disease 2019 (COVID19) due to SARS-CoV-2 in Hong Kong. Infect Control Hosp Epidemiol 2020;41:493-498.

5. Ng K, Poon BH, Kiat Puar TH, et al. COVID-19 and the risk to healthcare workers: a case report. Ann Intern Med 2020;172:766-767.

6. Wee LE, Sim XYJ, Conceicao EP, et al. Containment of COVID-19 cases among healthcare workers: the role of surveillance, early detection, and outbreak management. Infect Control Hosp Epidemiol 2020 May 11 [Epub ahead of print]. doi: 10.1017/ice.2020.219.

7. Tan CC. SARS in Singapore-key lessons from an epidemic. Ann Acad Med Singapore 2006;35:345-349.

8. McMichael TM, Currie DW, Clark S, et al. Epidemiology of COVID-19 in a long-term care facility in King County, Washington. $N$ Engl J Med 2020;382:2005-2011.

9. Graham LA, Maldonado YA, Tompkins LS, Wald SH, Chawla A, Hawn MT. Asymptomatic SARS-CoV-2 transmission from community contacts in healthcare workers. Ann Surg 2020 (in press).

\title{
The prevalence of severe acute respiratory coronavirus virus 2 (SARS-CoV-2) IgG antibodies in intensive care unit (ICU) healthcare personnel (HCP) and its implications - a single-center, prospective, pilot study
}

\author{
Mohsin Sheraz Mughal MBBS, Ikwinder Preet Kaur MBBS, Chandler D. Patton MD, Nagy H. Mikhail MD, \\ Chairut Vareechon PhD and Kenneth M. Granet MD, FACP \\ Monmouth Medical Center, an affiliate of the RWJ/Barnabas Health System, Long Branch, New Jersey
}

To the Editor-Healthcare personnel (HCP), including practitioners, nursing staff, respiratory therapists, and the pronepositioning team caring for coronavirus disease 2019 (COVID-19) patients in the intensive care unit (ICU) are considered to have a high risk of exposure to severe acute respiratory syndrome coronavirus 2 (SARS-CoV-2). Most patients admitted to the ICU are severely sick and need to be intubated. High-risk procedures for droplet dispersion, including tracheal intubation and tracheostomy tube placement, can be performed in the ICU. ${ }^{1}$ In a community seroprevalence study in Los Angeles County, the prevalence of antibodies to SARS-CoV-2 was $4.65 \%{ }^{2}$ To our knowledge, no other study has addressed the prevalence of subclinical seroconversion of SARSCoV-2 among HCP in the ICU setting. In this study, we investigated the seroconversion of asymptomatic SARS-CoV-2 infection in ICU HCP exposed to critically ill COVID-19 patients.

Author for correspondence: Kenneth M. Granet, E-mail: Kenneth.Granet@rwjbh.org. Cite this article: Mughal MS, et al. (2021). The prevalence of severe acute respiratory coronavirus virus 2 (SARS-CoV-2) IgG antibodies in intensive care unit (ICU) healthcare personnel (HCP) and its implications-a single-center, prospective, pilot study. Infection Control \& Hospital Epidemiology, 42: 638-639, https://doi.org/10.1017/ice.2020.298

\section{Methods}

This single-center, prospective, pilot study was performed in an ICU at a teaching hospital, Monmouth Medical Center in Long Branch, New Jersey. It was approved by our institutional review board. All HCP caring for COVID-19 patients in the ICU setting from March 1, 2020, to April 30, 2020, were eligible for inclusion in the study. A cross-sectional survey questionnaire was utilized to collect demographic characteristics and to exclude HCP who (1) tested positive for SARS-CoV-2 by reverse transcriptasepolymerase chain reaction assay (RT-PCR), (2) had symptoms consistent with COVID-19, or (3) had COVID-19 exposure in a household setting. In total, 134 ICU HCP responded to the survey, and $121 \mathrm{HCP}$ were eligible for SARS-CoV-2-specific IgG antibody testing. Means and interquartile ranges (IQRs) were used for continuous variables. All participants provided written consent. Antibody testing was performed on the sera using a rapid immunochromatography test (STANDARD Q COVID-19 IgM/IgG Duo, SD Biosensor, Suwon-si, Korea) by lateral flow in a Clinical Laboratory Improvement Amendments certified (CLIA), high-complexity laboratory. The manufacturer's stated 
sensitivity and specificity for IgG, 15-21 days after symptoms onset are $96.2 \%$ and $96.6 \%$, respectively. Blood specimens were drawn from 2 weeks after the specified period commencing May 14, 2020, and ending May 19, 2020.

\section{Results}

Overall, 134 ICU HCP responded to the survey: 75\% were women, $47.73 \%$ were registered nurses, $9.85 \%$ were attending physicians, $26.52 \%$ were resident physicians, $6.82 \%$ were patient care assistants, $6.82 \%$ were respiratory therapists, $1.52 \%$ were technicians, and $0.76 \%$ were anesthetists. The mean age of the respondents was 39.2 years (IQR, 28-48.5). The mean duration of work was 29.3 days (IQR, 16.0-40.0). Of 134 ICU HCP eligible staff, 13 were excluded and 121 underwent SARS-CoV-2-specific IgG antibody testing. One individual tested positive and 1 test result was inconclusive due to a faulty test kit and was removed from the analysis. In this study, the prevalence of asymptomatic seroconversion was $0.83 \%$.

\section{Discussion}

Information about the prevalence of asymptomatic seroconversion of SARS-CoV-2 in HCP is limited. In a preliminary report released by the Centers for Disease Control and Prevention (CDC), nearly 9,282 HCP have contracted COVID-19, and 27 have died. ${ }^{3}$ Okba et $\mathrm{al}^{4}$ demonstrated that most PCR-confirmed SARS-CoV-2 patients seroconverted after 2 weeks of disease onset. ${ }^{4}$ Our study revealed a prevalence of $0.83 \%$, which indicates that seroconversion in ICU HCP was a rare event. These data indicate that proper education and utilization of personal protective equipment (PPE) is effective. ${ }^{5}$ Additionally, ventilated patients have less aerosolization and were housed in a negative-pressure environment in the ICU isolation rooms, which also may have been factors in avoiding transmission to HCP.
Our study has several limitations. It was conducted in a single-center ICU and did not include long-term clinical or laboratory follow-up. Studies with larger sample sizes in different healthcare settings would be useful to validate the clinical impact of our findings.

Acknowledgments. We acknowledge Dr Violet E Kramer MD and Dr Margaret $\mathrm{H}$ Eng $\mathrm{MD}$ for their assistance. We acknowledge Joann Wolfson DNP, MSN, CCRN, with critical care services and Joseph Jaeger, $\mathrm{DrPH}$, chief academic officer, as well as Ali Jaffery, for contributing to data collection. We acknowledge Barbara Mihelic for institutional review board support at Monmouth Medical Center, Long Branch, New Jersey.

Financial support. No financial support was provided relevant to this article.

Conflicts of interest. All authors report no conflicts of interest relevant to this article.

\section{References}

1. Tran K, Cimon K, Severn M, Pessoa-Silva CL, Conly J. Aerosol-generating procedures and risk of transmission of acute respiratory infections to healthcare workers: a systematic review. PLoS One 2012;7(4):e35797. doi: 10.1371/ journal.pone.0035797.

2. Sood N, Simon P, Ebner P, et al. Seroprevalence of SARS-Cov-2-specific antibodies among adults in Los Angeles County, California, on April 10-11, 2020. JAMA 2020. https://doi.org/10.1001/jama.2020.8279.

3. Burrer SL, De Perio MA, Hughes, MM, et al. Characteristics of healthcare personnel with COVID-19-United States, February 12-April 9, 2020. Morbid Mortal Wkly Rept 2020;69:477-481.

4. Okba NM, Müller MA, Li W, et al. Severe acute respiratory syndrome coronavirus 2-specific antibody responses in coronavirus disease 2019 patients. Emerg Infect Dis 2020;6(7). doi: 10.3201/eid2607. 200841.

5. Seto W, Tsang D, Yung R, et al. Effectiveness of precautions against droplets and contact in prevention of nosocomial transmission of severe acute respiratory syndrome (SARS). Lancet 2003;361:1519-1520.

\title{
A framework for nosocomial transmission of emerging coronaviruses
}

\author{
Seth D. Judson $\mathrm{MD}^{1}$ and Vincent J. Munster $\mathrm{PhD}^{2}$ (1) \\ ${ }^{1}$ Department of Medicine, University of Washington, Seattle, Washington and ${ }^{2}$ Laboratory of Virology, Division of Intramural Research, National Institute of \\ Allergy and Infectious Diseases, National Institutes of Health, Hamilton, Montana
}

To the Editor-Over the past 17 years, 3 coronaviruses have emerged and caused diseases with high case fatality rates. From the severe acute respiratory syndrome (SARS) epidemic of 2002-2003, to outbreaks of Middle East respiratory syndrome (MERS) since 2013, to the pandemic of coronavirus disease 2019 (COVID-19), coronavirus diseases have afflicted global communities. Nosocomial, or healthcare-associated infections, have been recognized with each of these diseases because the viruses that cause these diseases are contagious, are relatively stable on surfaces, and are potentially disseminated through medical procedures.

\footnotetext{
Author for correspondence: Vincent J. Munster, E-mail: munstervj@niaid.nih.gov Cite this article: Judson SD and Munster VJ. (2021). A framework for nosocomial transmission of emerging coronaviruses. Infection Control \& Hospital Epidemiology, 42: 639-641, https://doi.org/10.1017/ice.2020.296
}

With the emergence of severe acute respiratory syndrome coronavirus 2 (SARS-CoV-2), the virus that causes COVID-19, many have wondered whether personal protective equipment (PPE) and hospital protocols are adequate to prevent transmission. To answer these questions, it is helpful to examine prior data for severe acute respiratory syndrome coronavirus 1 (SARS-CoV-1) and Middle East respiratory syndrome coronavirus (MERS-CoV).

Transmission of a virus occurs when an individual sheds viable virus that infects a susceptible host either through direct contact, through indirect contact with a contaminated surface (fomite transmission), or by exposure to virus-laden particles suspended in air. These particles are aerosols, which are often divided by size into large and small droplets. ${ }^{1}$ The term droplet transmission refers to infection via large droplets, and airborne transmission refers to 